\title{
ARTICLE OPEN \\ In situ control of graphene ripples and strain in the electron microscope
}

\author{
U. Ludacka ${ }^{1}$, M. R. A. Monazam ${ }^{1}$, C. Rentenberger (D) ${ }^{1}$, M. Friedrich ${ }^{2}$, U. Stefanelli ${ }^{2}$, J. C. Meyer (D) and J. Kotakoski (D)
}

We demonstrate control over the three-dimensional (3D) structure of suspended 2D materials in a transmission electron microscope. The shape of our graphene samples is measured from the diffraction patterns recorded at different sample tilts while applying tensile strain on the sample carrier. The changes in the shape of the pattern and in individual diffraction spots allow us to analyze both corrugations and strain in the lattice. Due to the significant effect of ripples and strain on the properties of 2D materials, our results may lead to new ways for their engineering for applications.

npj 2D Materials and Applications (2018)2:25; doi:10.1038/s41699-018-0069-z

\section{INTRODUCTION}

Graphene and other 2D materials differ from conventional bulk not only due to their extreme surface-to-bulk ratio and exotic properties resulting from quantum confinement of the electrons, but also because their structure can extend to the third available spatial dimension. This allows graphene to closely follow surface corrugations when supported on rough substrates ${ }^{1}$ and leads to a ripple structure in suspended graphene membranes. ${ }^{2,3}$ Curiously, the classical Mermin-Wagner Theorem ${ }^{4-6}$ excludes the possibility of realizing truly two-dimensional systems at finite temperature. Wavy patterns on the scale of approximately one hundred atom spacings have been computationally investigated ${ }^{3}$ and nonplanarity is expected also in the zero-temperature limit due to quantum fluctuations. ${ }^{7}$ Ripples are known to significantly influence the electronic ${ }^{8}$ and magnetic ${ }^{9}$ properties of graphene, and have even been argued to play a role in molecule permeation of this material. ${ }^{10}$ Hence, control over the ripple structure is important for the science and applications of graphene, as well as other 2D materials. In this work, we use transmission electron microscopy (TEM) to study ripples in as-prepared free-standing graphene, and further demonstrate that they can be controlled by applying uniaxial strain on the material in situ inside the microscope. In the direction of the applied strain, the ripples diminish below our detection limit, whereas changes in the perpendicular direction remain small. As a result, an aligned ripple structure is created, that could be used to introduce anisotropy for the charge and thermal transport in the material, paving way for new applications.

It is challenging to accurately measure corrugations in an atomically thin membrane. Although this can in principle be done with scanning probe techniques, in such studies graphene typically resides on a substrate and follows its surface topology. ${ }^{1}$ While this issue can be avoided by suspending graphene over small pits or trenches, also the interaction between the sample and the measurement device is known to influence the measured topography, ${ }^{11-14}$ except for very small suspended areas, ${ }^{15}$ where the graphene corrugations are caused by the confining geometry.
These issues can be overcome with TEM, where energetic electrons are passed through the sample to form its image. Unfortunately, due to the typical depth of field in TEM, sub-nmrange corrugations in a sample are not directly visible in real space images. However, electron diffraction in a TEM allows estimating the out-of-plane undulations in suspended 2D materials, as was shown for graphene in $2007^{2}$ and for molybdenum disulfide in 2011. ${ }^{16}$ The peak broadening in electron diffraction experiments provides a direct measure of the root mean square of the surface inclination, for which different experiments agree on values around $5^{\circ}{ }^{2,16,17}$ The amplitude of the out-of-plane corrugations of graphene was initially estimated to reach $1 \mathrm{~nm}$, whereas recent works suggest a root-mean-square roughness of $170^{17}$ or $114 \mathrm{pm}^{18}$ that can be further lowered down to $12 \mathrm{pm}$ via encapsulation with hexagonal boron nitride layers. ${ }^{18}$

In this article, we demonstrate in situ control of corrugations in freestanding graphene inside a transmission electron microscope. Our experimental results are supported through atomistic simulations, and a simple analytical model that allows us to discuss the relationship between the elastic moduli for in-plane stretch and bending, and confirms that the optimal configuration for graphene under our experimental conditions is not flat. We show that uniaxial pull can flatten the corrugations in the direction parallel to the pull leading to an aligned pattern of wrinkles instead of the originally randomly corrugated structure. After the flattening, graphene can be strained by continuous pulling. In our experiments the strain was limited to $\sim 1.5 \%$ due to the sample carrier that fractured at this point. The measured Poisson's ratio for graphene was 0.28 .

\section{RESULTS}

Analytical model

On the theoretical side, the non-flatness in graphene can be addressed by molecular mechanical methods, by analyzing optimal configurations in terms of given interaction energies. This is computationally well known $n^{3,7}$ and has been recently

\footnotetext{
${ }^{1}$ University of Vienna, Faculty of Physics, Boltzmanngasse 5, 1090 Vienna, Austria and ${ }^{2}$ University of Vienna, Faculty of Mathematics, Oskar-Morgernstern-Platz 1, 1090 Vienna, Austria

Correspondence: J Kotakoski (jani.kotakoski@univie.ac.at)
} 

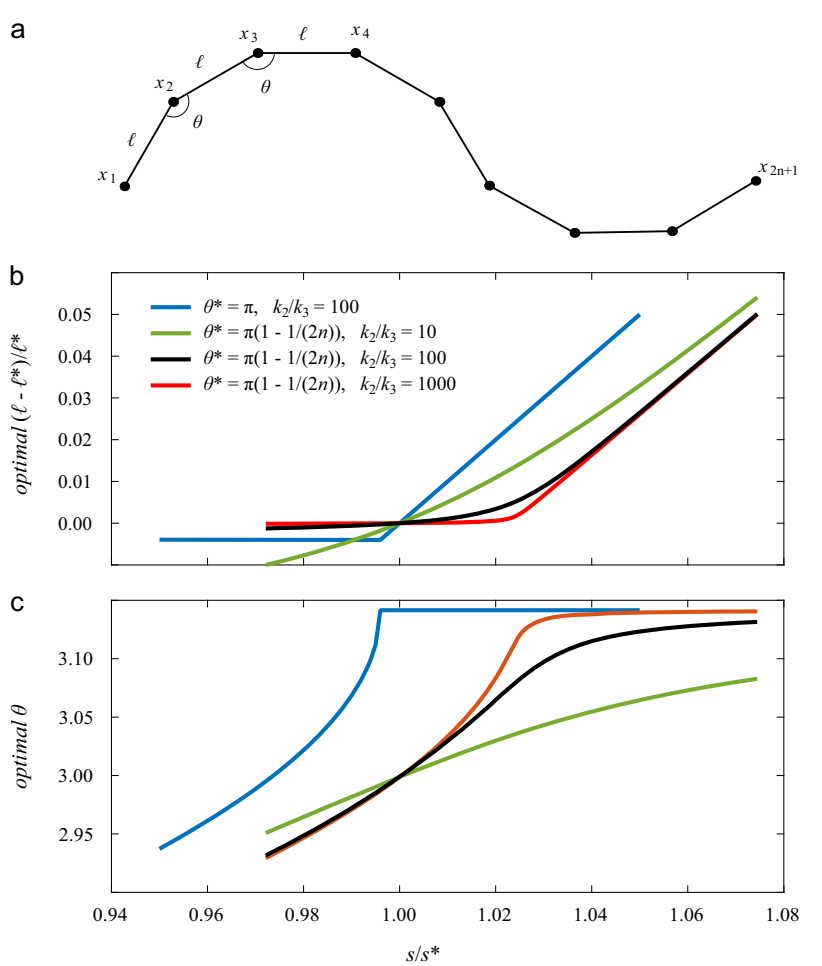

Fig. 1 Analytical model for a corrugated wave of locations. a An elementary wave consisting of $2 n+1$ locations (corresponding to hexagons in graphene). b Optimal (energy minimizing) strain $\varepsilon=$ $\left(\ell-\ell^{*}\right) / \ell^{*}$ in the chain as a function of applied stretching $s / s^{*}$. c Optimal (energy minimizing) corrugation angle $\theta$ as a function of applied stretching. For all simulations, $n=11, m=1$ and $\ell^{*}=1$.

analytically confirmed ${ }^{19,20}$ for a large class of configurational energies. By taking into account two-body interactions over a finite range of neighboring atoms, including at least second neighbors and three-body interactions, it has been shown that optimal configurations are indeed not flat. ${ }^{19}$ More precisely, whenever fixed at the boundary, such configurations show ripples with the same orientation having a specific wave-length independent of the size of the sample. ${ }^{20}$ These analytical results entail that the whole geometry of the optimal configuration is determined by one of its sections in the direction of the ripple. The 3D optimization problem can hence be efficiently rewritten in terms of a one-dimensional energy minimization problem for the configurational energy $E$ of a chain of locations $\left(x_{i}\right)_{i=1}^{2 m n+1}$ in $\mathbb{R}^{2}$, where the locations correspond to single hexagons of the abovementioned section, ${ }^{19} \mathrm{~m}$ stands for the number of waves and $2 n+$ 1 is the number of locations in each wave. One can also assume all pairs of adjacent locations $x_{i}$ and $x_{i+1}$ to have the same distance $\ell=\left|x_{i+1}-x_{i}\right|$ and all pairs of consecutive segments $x_{i+1}-x_{i}$ and $x_{i-1}-x_{i}$ to form the same angle $\theta$. Hence, the whole configuration is composed by $m$ waves of the type in Fig. $1 \mathrm{a}$.

A basic choice for the configurational energy $E$ is

$$
E(\ell, \theta)=2 m n k_{2}\left(\ell-\ell^{*}\right)^{2}+(2 m n-1) k_{3}\left(\theta-\theta^{*}\right)^{2}
$$

where the reference distance $\ell^{*}$ between adjacent locations, the reference angle $\theta^{*} \leq \pi$, and the parameters $k_{2}, k_{3}>0$ are given $\left(k_{2}\right.$ is related to the elastic modulus of the in-plane strain, whereas $k_{3}$ arises from the bending modulus). The coefficients in front of the bond and angle part of the energy express the fact that the configuration contains $2 m n$ bonds and $2 m n-1$ angles, respectively.

This model allows us to analytically investigate the optimal response of the wavy structure to external stretching by adjusting the bond length $\ell$ and the bending angle between adjacent locations (hexagons in graphene) $\theta$. To achieve this, let us investigate minimizers of $E$ among chains under prescribed stretching $s=\left|x_{2 m n+1}-x_{1}\right|$. Elementary trigonometry entails that

$s=2 m \ell \frac{\sin (n(\pi-\theta) / 2)}{\tan ((\pi-\theta) / 2)}$.

Given $s$, the latter relation enables us to express $\ell$ in terms of $\theta$. This reduces the dimension of the minimization problem and makes it amenable to numerical investigation. Figure $1 \mathrm{~b}$ shows the behavior of the normalized optimal bond strain $\left(\ell-\ell^{*}\right) / \ell^{*}$ as a function of the normalized stretching $s / s^{*}$. Here, $s^{*}$ is the length of the stress-free chain corresponding to $\ell=\ell^{*}$ and $\theta=\theta^{*}$. The normalization is such that $\left(\ell-\ell^{*}\right) / \ell^{*}=0$ for $s / s^{*}=1$. Figure $1 \mathrm{c}$ shows instead the optimal angle $\theta$ in terms of $s / s^{*}$.

Depending on the choice of the parameters, different behaviors arise. A first set of simulations assumes $\theta^{*}=\pi(2 n-1) /(2 n)$, corresponding to waves of aspect ratio wave-length/amplitude of approximately $10: 1$, in line with observations ${ }^{2}$ and computations. ${ }^{3}$ In this regime, small stretchings $\left(s / s^{*}>1\right)$ are accommodated by flattening the waves, so that $\left(\ell-\ell^{*}\right) / \ell^{*}$ remains close to 0 , being monotone increasing with $s / s^{*}$. For larger stretchings the optimal chain is almost flat $(\theta$ approaches $\pi$, see Fig. 1c) and the bond length increases. In particular, a linear behavior emerges. The ratio $k_{2} / k_{3}$ measures stretching vs. bending stiffness of the chain. By increasing this ratio, the response is progressively approaching a limiting piecewise affine behavior, with transition point at $\left(\ell-\ell^{*}\right) / \ell^{*}=0$ and $s / s^{*}=1$. Note that the value $k_{2} / k_{3}=10$ induces a strong monotonicity of the response under compression, which is not observed in our experiment (described below). Hence, one would rather assume $k_{2} / k_{3} \geq 100$.

By choosing $\theta^{*}=\pi$ one observes the onset of buckling under compression. Indeed, for mild compressions the bond length decreases and $\theta$ stays equal to $\pi$. Under larger compression the chain buckles and $\theta$ abruptly deviates from $\pi$. Correspondingly, the bonds elongate, giving rise to an antimonotone response. As this effect is not evident from our experiment, $\theta^{*}$ should rather be chosen strictly smaller than $\pi$ indicating a non-flat ideal configuration for graphene.

\section{Atomistic simulations}

Inspired by the theoretical results, we next turn to the experimental study of graphene ripples, assisted by atomistic simulations. The reciprocal representation of a flat graphene sample is a series of rods (Fig. 2a, b), that turn into cones for a corrugated membrane (Fig. 2C, d) due to a superposition of several diffracting beams corresponding to differently oriented lattice normals. At different sample tilts, the intersection of the Ewald sphere (approximated as a plane by the dotted lines in Fig. 2) cuts the cones at different heights, allowing one to establish a measure of the average surface inclination of the sample based on the measured size of the diffraction spot $(\sigma)$ as a function of the sample tilt (a). In Fig. 2e, we show the results of a linear fit for the $\sigma / a$ relationship for a set of simulated graphene samples with different values of $\gamma_{\text {rms }}$ (root mean square inclination). The fit to the data points is an interpolation that allows us to estimate the mean surface inclination from a measured $\sigma / a$ relationship up to $\sim 8^{\circ}$. Root-mean-square roughness $R_{\text {rms }}$ for the simulated structures varies between $75.2 \mathrm{pm}$ for the least corrugated membrane $\left(\gamma_{\mathrm{rms}} \approx 5.0^{\circ}\right)$ and $109.0 \mathrm{pm}$ for the most corrugated one $\left(\gamma_{\mathrm{rms}} \approx\right.$ $7.8^{\circ}$ ), which is close to the previously established values. ${ }^{17,18}$ The diffraction spot size $\sigma$ was estimated as the standard deviation of a (radially symmetric) 2D Gaussian peak fitted into the simulated diffraction spot. This way, the broadening of the diffraction spots can be directly related to the surface inclination. Another way to estimate the out-of-plane shape of the material from the diffraction pattern is to measure the intensity of the diffraction spot as a function of $a^{17,18}$ which however does not allow 


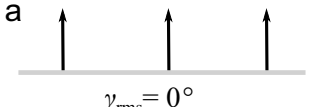

b
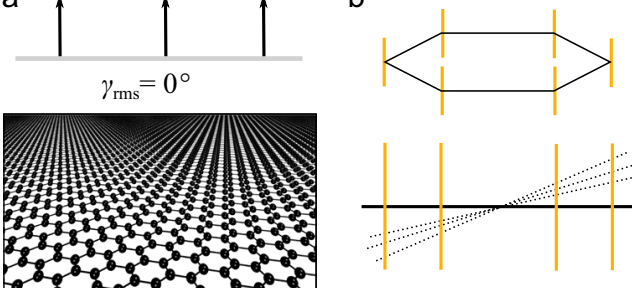

C

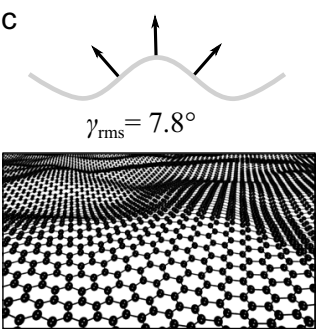

e

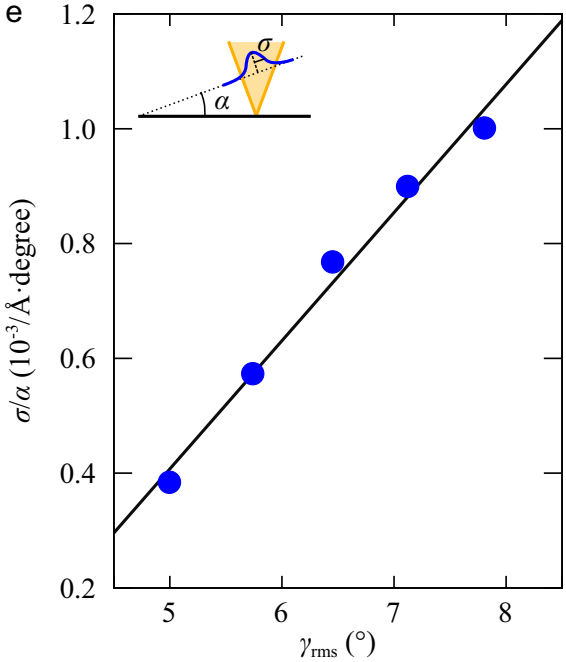

Fig. 2 Effect of varying surface inclination on the graphene diffraction pattern. Flat sample has parallel lattice normals throughout the sample $\mathbf{a}$, which leads to sharp rods in the reciprocal space $\mathbf{b}$ giving the same width for diffraction spots regardless of the sample tilt. In contrast, a corrugated sample $\mathbf{c}$ (here with root mean square inclination of $\gamma_{\mathrm{rms}}=7.8^{\circ}$ ) exhibits cone-like volumes in the reciprocal space $\mathbf{d}$ that lead to increasing diffraction spot sizes for higher sample tilts. e Relationship between the diffraction spot size $(\sigma)$ and the sample tilt $(a)$ for simulated corrugated graphene structures with varying values of $\gamma_{\mathrm{rms}}$. The solid line is a fit to the data points
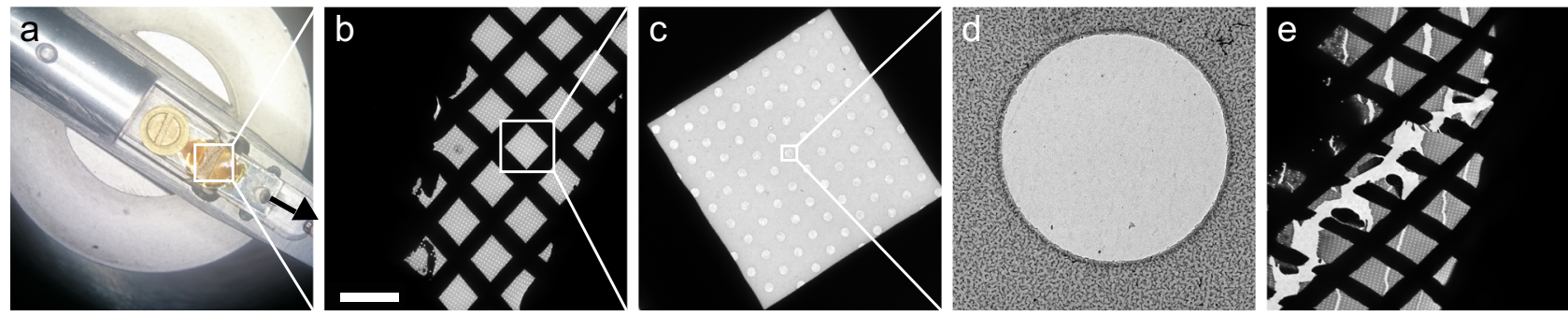

Fig. 3 Experimental setup. a Sample attached to two Al plates, mounted onto the tensile testing TEM holder. During the experiment, one of the Al plates is pulled step-by-step in the direction of the arrow, leading to stretching of the sample. $\mathbf{b}$ TEM image of the sample area between the two areas attached to the plates with an adhesive. The scale bar is $100 \mu \mathrm{m}$. c TEM image of a square within the Au TEM sample carrier, showing the sample-supporting amorphous carbon film with several holes. $\mathbf{d}$ TEM image of mono-layer graphene suspended over a hole in the support film. e TEM image of the sample area after the experiment showing a fractured sample carrier

distinguishing corrugations in the different in-plane directions, as is done below.

\section{In situ TEM experiments}

We started our experiments by measuring the $\sigma / a$ relation for suspended samples, similar to ref. ${ }^{2}$. Our experimental setup is illustrated in Fig. 3. The rippling in the initial samples appears random, and sometimes favors an arbitrary orientation, that is manifested as an asymmetry within the diffraction spots at higher $a$. An estimate for the initial $\gamma_{\mathrm{rms}}$ in our suspended CVD graphene samples, taking into account only those samples without preferred corrugation orientation, can be obtained from Fig. 2 using the average value of $\sigma / \alpha \approx 0.71 \times 10^{-3}$ (Å degree) $)^{-1}$, yielding $\gamma_{\mathrm{rms}} \approx$ $6.35^{\circ}$, which is almost identical to the slope estimated from ref. ${ }^{2}$ for mechanically exfoliated graphene, indicating that rippling does not significantly depend on the sample growth and preparation.

After establishing the initial shape of the sample, we moved onto applying uniaxial strain onto the sample carrier to measure its influence on graphene corrugations. The sample carrier was strained gradually with a step size of $0.25 \mu \mathrm{m}$ (less than $0.1 \%$ ). This strain of the sample carrier translates into stretching of graphene, analogous to the stretch discussed in the context of the analytical model. Typically, the manipulation became noticeable only after several steps with no apparent change in the sample (that is, after the pin of the stretching holder made a contact with the edge of the hole in the aluminium plate). Diffraction patterns were recorded over one hole in the support grid (with a spot size of about $\sim 0.5 \mu \mathrm{m}$ ) after every step, and additionally a tilting series in the range of $a \in\left[0^{\circ}, 27^{\circ}\right]$ was recorded after every four steps for every $\Delta a=3^{\circ}$. Sample areas were selected for the experiment so that they only displayed one set of diffraction peaks (only one crystalline orientation) and contained no folds or defects visible in the TEM images. All studied sample areas were mono-layer graphene. The experiment was continued until the amorphous carbon support fractured, which happened always much before a critical strain for the graphene membrane could be reached. At this point graphene typically tore at the edge of the suspended area. We did not notice any slippage during the experiments.

Assuming a rippled membrane that has a low bending rigidity, but that cannot be strained, this should lead to flattening of the membrane in the direction parallel to the pulling axis with no change or a slight increase in $\gamma_{\mathrm{rms}}$ in the perpendicular direction. Such flattening leads to increased projected lattice spacing, manifested as a decrease in the corresponding direction of the diffraction pattern. For increasing $\gamma_{r m s}$ the effect is opposite. In total, these effects should lead to progressively narrowing diffraction pattern. Three example diffraction patterns, recorded at different stages of the experiment, are shown in Fig. $4 a-c$.

Although not immediately clear from the patterns, they show exactly this behavior, as seen by analyzing their shape by fitting an ellipse into each diffraction pattern and observing both its 
a

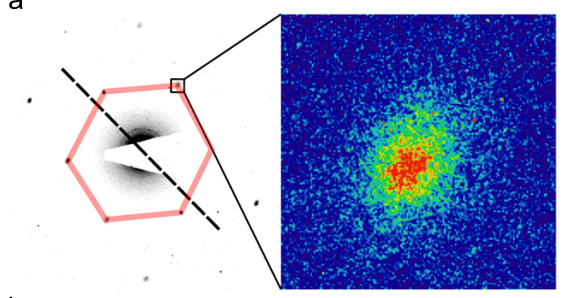

b

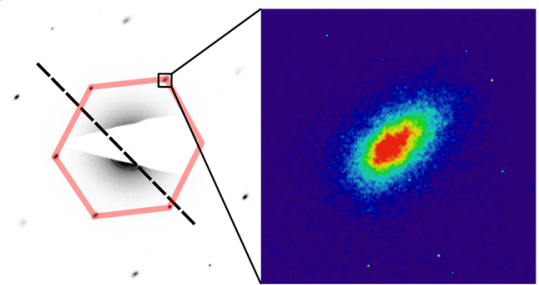

C

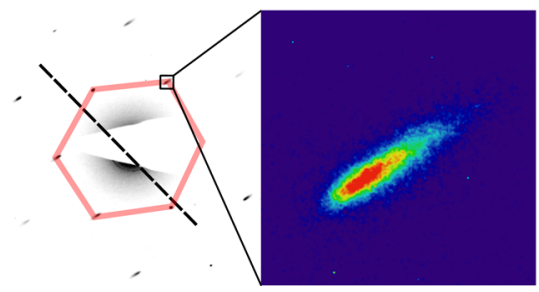

d

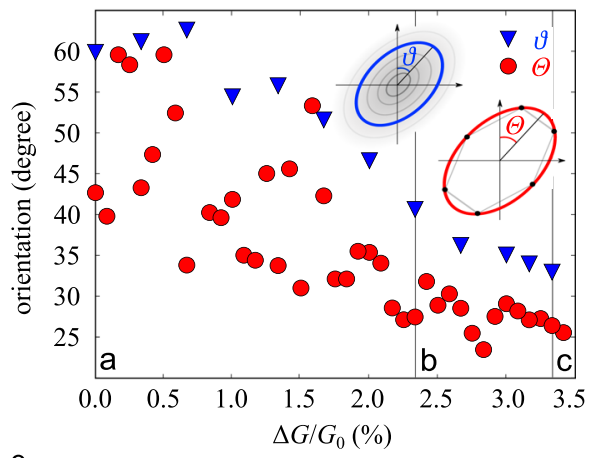

e

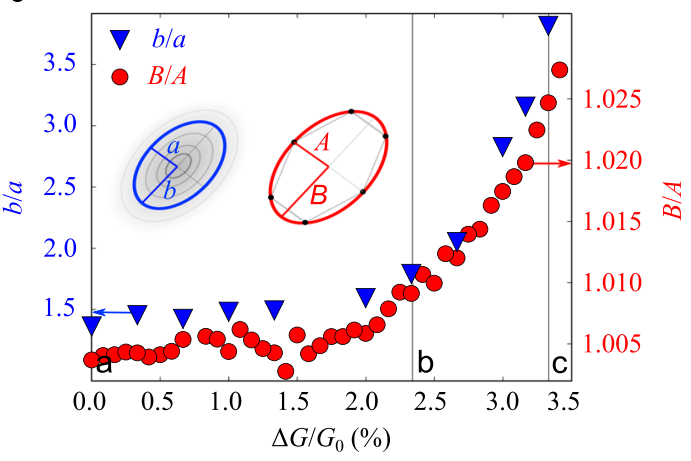

Fig. 4 Evolution of the diffraction pattern and individual diffraction spots during the experiment. a-c Diffraction pattern recorded at different stages of the experiment: $\mathbf{a}$ at the beginning, $\mathbf{b}$ towards the end, and $\mathbf{c}$ at the end. All shown patterns were recorded at sample tilt $a=21^{\circ}$. The dashed lines show the approximate tilt axis and the overlaid hexagons highlight the first set of diffraction peaks. The panels on the right show a zoom-in of the indicated diffraction spots in false color. $\mathbf{d}$ Orientation of the ellipse fitted to the diffraction patterns $(\Theta)$ and that of the diffraction spots $(\theta)$ during the experiment. e Ellipticity of the diffraction patterns ( $B / A$, right $y$-axis) and spots (b/a, left $y$-axis). Standard errors from the fits to the measured values are contained within the markers. All diffraction pattern values are for $a=0^{\circ}$ and spot values for $a=21^{\circ}$. In $\mathbf{d}$ and $\mathbf{e}$, the values corresponding to the diffraction patterns shown in a-c are marked with corresponding labels. The $x$-axis values $\left(\Delta G / G_{0}\right)$ indicate the relative change in the size of the gap in the sample carrier over which the sample was suspended

orientation and ellipticity (ratio between the major axis $B$ and the minor axis $A$ ). We stress that the change in orientation does not correspond to the rotation of the lattice during the experiment (the orientation of the diffraction pattern remains unchanged). Instead, it is related to the rotation of the corrugation pattern in the suspended graphene membrane that leads to a shear-like deformation of the diffraction pattern revealed by the orientation change of the ellipse. Figure $4 d$ shows the orientation of the diffraction pattern $(\Theta)$ with respect to the vertical axis of the image throughout the experiment (red circles). Towards the end of the experiment, the diffraction pattern begins to align itself at $25^{\circ}$ due to the continuous stretching of the sample carrier, revealing the direction of the deformation. The corresponding ellipticity values (Fig. $4 \mathrm{e}$, red circles) show an approximately linear increase between the diffraction patterns shown in Fig. 4b, c (between the two vertical lines in Fig. 4d, e). However, even before this an increase is apparent in the ellipticity of the diffraction pattern, presumably arising from the change in apparent lattice spacing in the direction of the pull.

Due to the asymmetry introduced through stretching, a radially symmetric 2D Gaussian peak shape cannot be used to describe the peaks during this experiment. Instead, we use the generalized Gaussian peak shape with two orthogonal standard deviations in an orientation that is individually matched to each measured diffraction spot. Overall, the spots show very similar behavior to the diffraction patterns, also indicating alignment towards the pull direction (Fig. 4d, blue triangles) and changes in the out-of-plane structure of the membrane (Fig. 4e, blue triangles). However, despite the qualitatively similar behavior, the measured ellipticity values for the diffraction spots increase significantly more than those for the diffraction patterns due to the different underlying physical mechanisms.

Separately measured diffraction spot sizes $(\sigma$, standard deviations of the 2D Gaussian peak) for the directions parallel and orthogonal to the pull direction allow us to study in detail how the stretching of the sample carrier changes the corrugations in graphene. As can be seen in Fig. 5, for some samples the generalized peak shape reveals an asymmetry in the diffraction spots already at the beginning of the experiments. However, at this point the axis of asymmetry is arbitrary (ca. $60^{\circ}$ for this sample, as seen from Fig. 4d). At the beginning of the experiment, the axis rotates to align with the direction of the mechanical force applied to the sample carrier up to a relative increase in width (i.e., stretching) of $\Delta G / G_{0} \approx 2.0 \%$. At the same time, $\gamma_{\mathrm{rms}}$ in both observed directions remains nearly constant. This leads to the plateau in the ellipticity values in Fig. 4e. After this point, the change in the orientation of the spot (Fig. 4d) becomes more gradual and the ellipticity starts to increase rapidly (Fig. 4e).

In Fig. $5 a$, the changes in the $\sigma / a$ values, and correspondingly, the mean inclinations, for the two in-plane directions are plotted separately for the minor and the major axis of the diffraction spot. Since only the values corresponding to the short axis change during the experiment, the increase of the ratio $b / a$ observed in Fig. $4 \mathrm{e}$ is solely due to a flattening in direction parallel to the pull axis (corresponding to the narrower direction of the diffraction spot). Towards the end of the experiment, $\gamma_{\text {rms }}$ parallel to the pull axis becomes so small that it falls below our detection limit ( $\sigma$ at all $a$ become similar in size to those of non-tilted samples, that is, smaller than the intrinsic peak width determined by our experimental setup). 

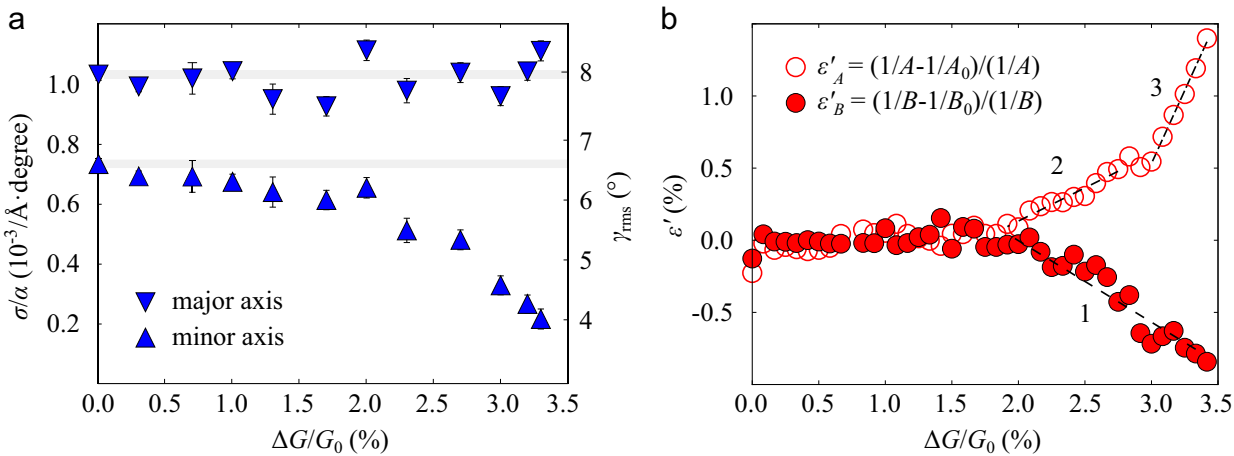

Fig. 5 Effect of stretching the sample carrier on the out-of-plane corrugation and strain in suspended graphene. a Changes in the maximum (major axis) and minimum $\gamma_{\mathrm{rms}}$ (minor axis) in graphene during the experiment ( $\gamma_{\mathrm{rms}}$, right $y$-axis), as estimated from the $\sigma / a$ values (left $y$-axis) measured from diffraction spots. Error bars show the standard errors from the fits to the experimental data and horizontal gray lines are guides to the eye. $\mathbf{b}$ Apparent strain in real space in two orthogonal directions, as estimated from the diffraction patterns at zero tilt ( $a=0$ ) during the experiment. Initial axes lengths $A_{0}$ and $B_{0}$ are estimated as the averages of the first 15 values for each data set, and the lines marked with labels $1-3$ are fits to the corresponding data points with following slopes: (1) $-0.57 \pm 0.05,(2) 0.46 \pm 0.03$, and (3) $2.02 \pm 0.09$ (uncertainties are standard errors of the fits). The $x$-axis values $\left(\Delta G / G_{0}\right)$ indicate the relative change in the size of the gap in the sample holder over which the sample was suspended

Finally, in Fig. 5b we show the apparent strain in the graphene lattice as estimated from the diffraction patterns in two orthogonal directions. In agreement with the previously discussed data, no difference in the lattice spacing is observed in the diffraction pattern until a stretch of $\Delta G / G_{0} \approx 2.0 \%$, despite the rotation of the corrugation pattern (as observed from Fig. 4d). After this point the ellipticity of the diffraction pattern starts to increase (Fig. 4e), which based on Fig. $5 \mathrm{~b}$ can be associated with simultaneous changes in both observed orthogonal directions. Until a stretch of $\Delta G / G_{0} \approx 3.0 \%$ both axes behave similarly (albeit in opposite directions), which is clearly observed by the slopes of the lines fitted to the calculated apparent strain ${ }^{21}$ (Fig. 5b) within this range $(-0.57 \pm 0.05$ vs. $0.46 \pm 0.03)$. In other words, while the graphene membrane is stretched in one direction it shrinks in the other, with an exceptionally high positive Poisson's ratio of $-(-0.57) / 0.46=1.24$. However, this ratio is maintained only over a small range of stretching and concurs with the continued alignment of the overall corrugation pattern from meandering landscape of loosely oriented hills and valleys into an aligned set of wrinkles. However, after $\Delta G / G_{0} \approx 3.0 \%$, a clear change occurs leading to a much steeper increase of the lattice spacing in the direction of the pull (increase of the apparent strain with a slope of $2.03 \pm 0.09$ in Fig. 5b). Hence, it is a clear indication of applied uniaxial strain within the graphene lattice. The corresponding Poisson's ratio is 0.28 . The discontinuity seen in Fig. $5 \mathrm{~b}$ is common for all of our samples. The slope value $>1$ at the end of the experiment is attributed to a non-linear strain transfer from the sample carrier to the sample, likely caused by a partial fracture in the carrier. This allows us at the end of the experiment to measure the intrinsic properties of graphene itself.

\section{DISCUSSION}

We can directly compare our experimental results (Fig. 5) against those obtained with the analytical model (Fig. 1). The experimentally observed change in the mean curvature $\left(\gamma_{\mathrm{rms}}\right)$ is directly related to the angle of curvature in the model $(\theta)$, and the apparent measured strain $\varepsilon^{\prime}$ corresponds to $\varepsilon=\left(\ell-\ell^{*}\right) / \ell^{*}$, whereas the applied (uniaxial) stretchings are directly equivalent. From the experimental results, it is clear that flattening of graphene continues beyond the point where the material starts to get strained $\left(\varepsilon^{\prime}>0\right)$, confirming the analytical result indicating that $\theta^{*}<\pi$, i.e., the optimal configuration for graphene is not flat, ${ }^{19}$ at least under these experimental conditions. The experiments further show that the strain in graphene reaches $\sim 1 \%$ for stretching of ca. $3.25 \%$, which sets a lower limit for the $k_{2} / k_{3}$ ratio to $\geq 100$. Further looking at the reached $\gamma_{\text {rms }}$ at this point, it is clear that $\theta$ is not yet close to $\pi$, which further limits the $k_{2} / k_{3}$ to $<1000$, providing a direct experimental order-of-magnitude estimate for the ratio of the elastic moduli for in-plane stretch $\left(k_{2}\right)$ and bending $\left(k_{3}\right): 100 \leq k_{2} / k_{3}<1000$.

The highest obtained strains in our experiments were ca. $1.5 \%$, after which the sample carrier fractured, which in some cases lead to tearing of the graphene at the rim of the suspended area. For most samples we could also record a tilt series after this, which revealed again a randomly corrugated graphene membrane with $\gamma_{\text {rms }}$ values close to as-prepared samples. This shows that, given a suitable sample carrier, the observed changes (alignment of the ripples, flattening of the membrane and uniaxial strain) are reversible.

In conclusion, we have shown that the three-dimensional (3D) structure of suspended graphene can be controlled reversibly by stretching the sample carrier. This leads to a variety of gradual changes first in the intrinsic corrugations of graphene and later into straining of the graphene lattice along the direction of pull. At the initial stages, the initially randomly oriented corrugation pattern starts to align itself according to the pull direction, that can easily be seen by observing the shear-like deformation of the diffraction pattern. After the orientational alignment, continuous stretching leads to flattening of graphene in the direction parallel to the pull direction down to such a low average inclination that it disappears below our detection limit, creating a pattern of aligned wrinkles. At the final stages of the process, when the corrugation pattern cannot change any more, continuous stretching leads to straining of the lattice. In our experiments, that relied on commercial graphene samples using standard electron microscopy grids as sample carriers, only moderate amounts of strain $(\sim 1.5 \%)$ could be realized before the support material fractured. With specifically designed support solutions significantly higher strains should be within the reach and allow a large variety of studies on the influence of corrugations and strain on 2D materials inside the transmission electron microscope. Due to the significant influence of out-of-plane structure of graphene and other 2D materials to their properties, by providing direct control over corrugations and strain, this work can also be expected to open the way for new applications. Due to the different phonon and electron scattering for flat and corrugated graphene structures, one could use this method for creating graphene-based structures with spatially anisotropic thermal and electronic conduction determined by the pattern of corrugations. 


\section{METHODS}

\section{Simulations}

For atomistic simulations, graphene samples were created using 24,000 carbon atoms. In order to create the corrugation into graphene, the model was strained and heated up for $5 \mathrm{~ns}$ to $6 \mathrm{~K}$ and cooled down for $8 \mathrm{~ns}$ to $0.1 \mathrm{~K}$ and partially relaxed at the end. Diffraction patterns were calculated for five different states of compressed graphene, where the compression was partially released in each step. The simulations as well as the electron diffraction pattern calculations were performed with large-scale atomic/ molecular massively parallel simulator (LAMMPS). ${ }^{22-24}$ Although $\sigma$ does depend on the distance between the diffraction spot and the origin of the reciprocal space, it has only a minor influence on the $\sigma / a$ relationship, which is used here to estimate the root mean square inclination $\gamma_{\text {rms. }}$. The interaction between carbon atoms were treated using the LCBOP potential. ${ }^{25}$ The inclination was estimated from the surface normals calculated for the atomic structure divided into triangles defined by each carbon atom and two of its neighbors.

\section{Experiments}

Our samples were commercial chemical-vapor-deposition (CVD)-grown graphene suspended on gold-supported Quantifoil ${ }^{T M}$ amorphous carbon grids with ca. $2 \mu \mathrm{m}$ holes purchased from Graphenea (TM). The grids were individually attached with an adhesive (epoxy with conductive paste to reduce charging in the TEM) to two small aluminium plates separated by a gap of $G_{0} \approx 300 \mu \mathrm{m}$. The plates were then placed on a in situ tensile testing TEM holder (Philips PW 6552) and inserted into the microscope (see Fig. 3). The study was performed with a Philips CM200 microscope at $80 \mathrm{kV}$ to reduce the knock-on damage at the sample. ${ }^{26}$ Astigmatism in the diffraction patterns was minimized throughout the experiment.

\section{Data availability}

Original data including recorded diffraction patterns, atomistic simulation structures, and simulated diffraction patterns are available through Phaidra, the digital assets repository of University of Vienna, at http:// phaidra.univie.ac.at/o:803015. Analyzed data and computer programs used for analysis are available from the corresponding author on reasonable request.

\section{ACKNOWLEDGEMENTS}

U.L., M.F., U.S., and J.K. acknowledge funding from the Wiener Wissenschafts-, Forschungs- und Technologiefonds (WWTF) via project MA14-009, M.R.A.M and J.K. from the Austrian Science Fund (FWF) through project 13181-N36, C.R. through project I1309, J.C.M. through project P25721-N20 and U.L. through F65, P27052, and 12375. M.F. acknowledges the support from Alexander von Humboldt Foundation. We also acknowledge Vienna Scientific Cluster for generous grants of computational resources.

\section{AUTHOR CONTRIBUTIONS}

U.L. carried out the experimental work with the assistance of C.R. Analytical model was developed by M.F. and U.S., who also performed the related simulations. Atomistic simulations were carried out by M.R.A.M. The study was conceived by J.C.M. and J.K. J.K. supervised the experimental and computational work. U.L. and J.K. analyzed the data and wrote the article based on contributions from all authors.

\section{ADDITIONAL INFORMATION}

Competing interests: The authors declare no competing interests.

Publisher's note: Springer Nature remains neutral with regard to jurisdictional claims in published maps and institutional affiliations.

\section{REFERENCES}

1. Ishigami, M., Chen, J. H., Cullen, W. G., Fuhrer, M. S. \& Williams, E. D. Atomic structure of graphene on $\mathrm{SiO}_{2}$. Nano Lett. 7, 1643-1648 (2007).
2. Meyer, J. C. et al. The structure of suspended graphene sheets. Nature $\mathbf{4 4 6}, 60-63$ (2007).

3. Fasolino, A., Los, J. H. \& Katsnelson, M. I. Intrinsic ripples in graphene. Nat. Mater. 6, 858-861 (2007).

4. Landau, L. D. \& Lifshitz, E. M. Statistical Physics (Pergamon, Oxford, 1980).

5. Mermin, N. D. Crystalline order in two dimensions. Phys. Rev. 176, 250-254 (1968).

6. Mermin, N. D. \& Wagner, H. Absence of ferromagnetism or antiferromagnetism in one- or two-dimensional isotropic heisenberg models. Phys. Rev. Lett. 17, 1133-1136 (1966).

7. Herrero, C. P. \& Ramirez, R. Quantum effects in graphene monolayers: pathintegral simulations. J. Chem. Phys. 145, 224701 (2016).

8. Vasić, B., Zurutuza, A. \& Gajić, R. Spatial variation of wear and electrical properties across wrinkles in chemical vapour deposition graphene. Carbon N. Y. 102, 304-310 (2016).

9. Schiefele, J., Martin-Moreno, L. \& Guinea, F. Faraday effect in rippled graphene: magneto-optics and random gauge fields. Phys. Rev. B 94, 035401 (2015).

10. Liang, T. et al. Permeation through graphene ripples. 2D Mater. 4, 025010 (2017).

11. Zan, R. et al. Scanning tunnelling microscopy of suspended graphene. Nanoscale 4, 3065-3068 (2012).

12. $\mathrm{Xu}, \mathrm{P}$. et al. Atomic control of strain in freestanding graphene. Phys. Rev. B $\mathbf{8 5}$, 121406 (2012).

13. Eder, F. R. et al. Probing from both sides: reshaping the graphene landscape via face-to-face dual-probe microscopy. Nano Lett. 13, 1934-1940 (2013).

14. Breitwieser, R. et al. Flipping nanoscale ripples of free-standing graphene using a scanning tunneling microscope tip. Carbon N. Y. 77, 236-243 (2014).

15. Tapasztó, L. et al. Breakdown of continuum mechanics for nanometrewavelength rippling of graphene. Nat. Phys. 8, 739-742 (2012).

16. Brivio, J., Alexander, D. T. L. \& Kis, A. Ripples and layers in ultrathin MoS2 membranes. Nano Lett. 11, 5148-5153 (2011).

17. Kirilenko, D. A., Dideykin, A. T. \& Tendeloo, G. V. Measuring the corrugation amplitude of suspended and supported graphene. Phys. Rev. B 84, 235417 (2011).

18. Thomsen, J. D. et al. Suppression of intrinsic roughness in encapsulated graphene. Phys. Rev. B 96, 014101 (2017).

19. Friedrich, M. \& Stefanelli, U. Graphene ground states. Preprint at https://arxiv.org/ abs/1802.05049 (2018)

20. Friedrich, M. \& Stefanelli, U. Ripples in graphene: a variational approach. Preprint at https://arxiv.org/abs/1802.05053 (2018).

21. Ebner, C., Sarkar, R., Rajagopalan, J. \& Rentenberger, C. Local, atomic-level elastic strain measurements of metallic glass thin films by electron diffraction. Ultramicroscopy 165, 51-58 (2016).

22. Plimpton, S. Fast parallel algorithms for short-range molecular dynamics. J. Comput. Phys. 117, 1-19 (1995).

23. Plimpton, S. J. \& Thompson, A. P. Computational aspects of many-body potentials. MRS Bull. 37, 513-521 (2012).

24. Coleman, S. P., C, L. \& Spearot, D. E. Virtual diffraction analysis of ni [010] symmetric tilt grain boundaries. Model. Simul. Mater. Sci. Eng. 21, 055020 (2013).

25. Los, J. H. \& Fasolino, A. Intrinsic long-range bond-order potential for carbon: performance in Monte carlo simulations of graphitization. Phys. Rev. B 68, 024107 (2003).

26. Susi, T. et al. Isotope analysis in the transmission electron microscope. Nat. Commun. 7, 13040 (2016).

Open Access This article is licensed under a Creative Commons Attribution 4.0 International License, which permits use, sharing, adaptation, distribution and reproduction in any medium or format, as long as you give appropriate credit to the original author(s) and the source, provide a link to the Creative Commons license, and indicate if changes were made. The images or other third party material in this article are included in the article's Creative Commons license, unless indicated otherwise in a credit line to the material. If material is not included in the article's Creative Commons license and your intended use is not permitted by statutory regulation or exceeds the permitted use, you will need to obtain permission directly from the copyright holder. To view a copy of this license, visit http://creativecommons. org/licenses/by/4.0/.

(c) The Author(s) 2018 